\title{
Nutritional intervention in patients with hypopituitarism secondary to pituitary adenomas with metabolic syndrome
}

\author{
Renata Elisabeth Rothen*, Bruna Breyer de Freitas, Carolina Garcia Soares Leães, Miriam da Costa Oliveira, \\ Fernanda Michielin Busnello, Júlia Fernanda Semmelmann Pereira-Lima
}

From 20th Brazilian Diabetes Society Congress

Porto Alegre, Brazil. 11-18 November 2015

\section{Background}

The deficiency in the production of any of the pituitary hormones, denominated hypopituitarism, may cause the increase of visceral fat, dyslipidemia and decrease of muscle mass. Metabolic syndrome (MetS) is a complex disorder, characterized by alterations of the lipid profile, abdominal obesity, blood pressure and insulin resistance, being highly prevalent in hypopituitarism.

\section{Objective}

We performed clinical, laboratory and nutritional assessment in patients with hypopituitarism, pituitary adenomas and MetS, before and six months post nutritional intervention and compared outcomes.

\section{Materials and methods}

this was a cross-sectional study of 36 outpatients, aged 20-75 yrs., with MetS whose diagnosis was established based on the International Diabetes Federation criteria and hypopituitarism, in the presence or after pituitary adenoma treatment. In the anthropometric assessment the body weight, height, body mass index (BMI) and waist circumference (WC) were measured. Furthermore, serum lipid levels and fasting glucose were measured. Nutritional assessment included sociodemographic information, historic of diseases, medications as well as food intake by a $24-\mathrm{h}$ food recall. These were evaluation prior and post nutritional intervention, on initial visit (V0) and final visit (V6) after six months.

* Correspondence: renataer@hotmail.com UFCSPA, Porto Alegre, Brazil original work is properly cited. The Creative Commons Public Domain Dedication waiver (http://creativecommons.org/publicdomain/ zero/1.0/) applies to the data made available in this article, unless otherwise stated. 